\title{
Mechanisms Mediating Antigenic Variation in Trypanosoma brucei
}

\author{
Gloria Rudenko
}

Wellcome Trust Centre for the Epidemiology of Infectious Disease, Department of Zoology, University of

Oxford, South Parks Road, Oxford OX1 3PS, England

\begin{abstract}
Antigenic variation in Trypanosoma brucei is a highly sophisticated survival strategy involving switching between the transcription of one of an estimated thousand variant surface glycoprotein (VSG) genes. Switching involves either transcriptional control, resulting in switching between different VSG expression sites; or DNA rearrangement events slotting previously inactive VSG genes into an active VSG expression site. In recent years, considerable progress has been made in techniques allowing us to genetically modify infective bloodstream form trypanosomes. This is allowing us to reengineer VSG expression sites, and look at the effect on the mechanisms subsequently used for antigenic variation. We can now begin a dissection of a highly complicated survival strategy mediated by many different mechanisms operating simultaneously.
\end{abstract}

Key words: antigenic variation - Trypanosoma brucei - variant surface glycoprotein (VSG) -

VSG expression site

\section{MECHANISMS OF ANTIGENIC VARIATION}

African trypanosomes are effective pathogens, as they can establish chronic infections in man and his lifestock, despite being fully exposed to periodic assault by the immune system. Their survival strategy is based on ensuring that at a low frequency, trypanosomes arise which have changed the epitopes exposed on their surface. This exchangeable surface coat is a homogeneous layer composed of a single variant surface glycoprotein (VSG). As each individual trypanosome encodes up to a thousand VSG genes, and is capable of making chimaeric new ones, the potential for variation during a protracted infection is endless.

Intense research by many laboratories in the eighties laid the basis for what is known about the main mechanisms of VSG switching in bloodstream form Trypanosoma brucei. Any given VSG coat is encoded by a single VSG gene transcribed in an expression site located at the ends of certain chromosomes. As there are 20 bloodstream form VSG expression sites, one way of switching is simply to silence the active expression site and activate a new one (an in situ switch). This method of switching accesses a relatively small pool of 20 VSG genes. However, recent evidence indicates that the different VSG expression sites each con-

Fax: +44-1865-281.245.

E-mail: gloria.rudenko@zoo.ox.ac.uk

Received 12 November 1998

Accepted 19 January 1999 tain genes encoding receptor proteins that are optimized for different hosts (Bitter et al. 1998). This could mean that in situ switches are important during the establishment of the trypanosome in a new host species.

VSG switching can also be mediated by DNA rearrangements like gene conversions or telomere exchange. Gene conversions access the largest pool of VSG genes (virtually all of them). A silent VSG gene is copied and inserted into the active expression site, replacing the old VSG gene. Alternatively, trypanosomes can switch via telomere exchange, whereby a silent VSG gene at a chromosome end is flipped into the active VSG expression site. Recent overviews of antigenic variation are Donelson (1995), Vanhamme and Pays (1995), Borst et al. (1996), Cross (1996), Barry (1997), Rudenko et al. (1998b).

The problem with studying antigenic variation in the field has been that all of these mechanisms are operating at the same time, and antigenic populations of parasites within an animal are normally not monoclonal. The $20 \mathrm{VSG}$ expression sites are highly similar with each other, with only the VSG gene as a unique marker. Once the VSG gene has been switched a few times, it can be very difficult to reconstruct how this has happened. Due to the polyclonal nature of the infection, it is also impossible to prove that trypanosomes present in various peaks are sequentially derived from each other. In contrast, single relapse experiments with laboratory strains are a highly simplified model system, which should give us insights into how antigenic variation is mediated in field strains. 


\section{NEW METHODS TO STUDY VSG EXPRESSION SITE SWITCHING}

Methods developed recently at the Rockefeller University in New York allow the cloning of infective bloodstream form trypanosomes on agarose plates (Carruthers \& Cross 1992) and their stable transfection (Carruthers et al. 1993). Cloned trypanosomes remain infective, and T. brucei populations cultured on agarose plates don't lose their pleomorphic phenotype (Vassella \& Boshart 1996). These techniques are allowing us to genetically modify VSG expression sites, and look at the subsequent effect on the mechanisms used to mediate antigenic variation in bloodstream form trypanosomes. I will give a number of examples where recent experiments are making us reevaluate old hypotheses about how VSG expression site switching occurs.

DNA transfection experiments that add or exchange promoters in expression sites, have shown us that VSG expression site control does not appear to require a specific promoter sequence. An rDNA promoter replacing the endogenous VSG expression site promoter can be switched on and off effectively (Rudenko et al. 1995). In addition, different non-homologous promoters inserted into various positions in the expression site are silenced essentially equally well (Horn \& Cross 1995, 1997a,b). These results were unexpected, as it had been assumed that the specific sequence of the VSG expression site promoter was recognized by specific transcription factors which played a role in switching. This possibility now appears to be unlikely.

During chronic infections a rough form of patterning is seen, as the switch from one VSG coat to another is not entirely random but can occur according to a rough pattern. It had been postulated that this ordered appearance of coats was due to the varying viabilities of different double expressing switch intermediates wearing different combinations of double VSG coats (Agur et al. 1989). Although this was an intriguing hypothesis, genetically modified trypanosomes have not supported it. Three different pairs of trypanosome strains reengineered to express a second additional VSG gene inserted in the active VSG expression site, though double VSG expressors, showed no growth disadvantage in rodents (Munoz-Jordan et al. 1996).

Lastly, all known VSG expression sites contain characteristic arrays of $70 \mathrm{bp}$ repeat sequences upstream of the telomeric VSG gene. A few of these repeats are also present upstream of chromosome internal basic copy VSG genes. It had been hypothesized that these $70 \mathrm{bp}$ repeat sequences were the targets of a sequence specific endonuclease which generated a cut, allowing the gene conversion of a new VSG gene. If this were the case, then deleting the $70 \mathrm{bp}$ repeat arrays from the active expression site should result in the drastic reduction of gene conversion reactions with the active expression site. This was shown not to be the case. Trypanosomes without $70 \mathrm{bp}$ repeat arrays in the active expression site that were allowed to switch their VSG coat, underwent as many gene conversion reactions as wild type trypanosomes.

As expected in these trypanosomes, the donor sequences were exclusively other expression sites rather than chromosome internal VSG genes (McCulloch et al. 1997). Therefore, no evidence was found that the 70 bp repeat sequences were a particular "hot spot" for gene conversion reactions.

\section{VSG EXPRESSION SITE ANALYSIS}

Genetic manipulation has also helped in the characterization of VSG expression sites. As the many VSG expression sites are almost identical to each other, selectable markers inserted into the promoter areas can be used as tags in the analysis of the expression site promoter region. Analysis of these marked expression sites after in situ switches have not shown detectable obligatory DNA rearrangements (Navarro \& Cross 1996, Horn \& Cross, 1997a, Rudenko et al. 1998a). In addition, the unique marker genes inserted have been used to probe the expression site promoter area for $\mathrm{J}$, a unique DNA modification found in trypanosomatids (Van Leeuwen et al. 1997).

Lastly, transfection experiments are being used to determine the function of the multiple expression site associated genes (ESAGs) present in the polycistronic VSG expression site transcription unit. Inactivating the ESAG1 gene in an active expression site, does not give a detectable phenotype (Carruthers et al. 1996). This could be due to the redundancy of multiple additional ESAG1 genes present in the genome.

In short, the capacity to modify the trypanosome genome in vitro has revolutionized our ability to dissect apart something as complicated as antigenic variation in African trypanosomes.

\section{REFERENCES}

Agur Z, Abiri D, van der Ploeg LHT 1989. Ordered appearance of antigenic variants of African trypanosomes explained in a mathematical model based on a stochastic switch process and immune-selection against putative switch intermediates. Proc Natl Acad Sci USA 86: 9626-9630.

Barry JD 1997. The relative significance of mechanisms of antigenic variation in African trypanosomes. Parasitol Today 13: 212-218.

Bitter W, Gerrits H, Kieft R, Borst P 1998. The role of transferrin-receptor variation in the host range of Trypanosoma brucei. Nature 391: 499-502. 
Borst P, Rudenko G, Taylor MC, Blundell PA, van Leeuwen F, Bitter W, Cross M, McCulloch R 1996. Antigenic variation in trypanosomes, Arch Med Res 27: 379-388.

Carruthers VB, Cross GAM 1992. High-efficiency clonal growth of bloodstream- and insect-form Trypanosoma brucei on agarose plates. Proc Natl Acad Sci USA 89: 8818-8821.

Carruthers VB, van der Ploeg LHT, Cross GAM 1993. DNA-mediated transformation of bloodstream-form Trypanosoma brucei. Nucl Acids Res 21: 2537-2538.

Carruthers VB, Navarro M, Cross GAM 1996. Targeted disruption of expression site- associated gene- 1 in bloodstream-form Trypanosoma brucei. Mol Biochem Parasitol 81: 65-79.

Cross GAM 1996. Antigenic variation in trypanosomes: secrets surface slowly. BioEssays 18: 283-291.

Donelson JE 1995. Mechanisms of antigenic variation in Borrelia hermsii and African trypanosomes. $J$ Biol Chem 270: 7783-7786.

Horn D, Cross GAM 1995. A developmentally regulated position effect at a telomeric locus in Trypanosoma brucei. Cell 83: 555-561.

Horn D, Cross GAM 1997a. Analysis of Trypanosoma bruce i vsg expression site switching in vitro. Mol Biochem Parasitol 84: 189-201.

Horn D, Cross GAM 1997b. Position-dependent and promoter-specific regulation of gene expression in Trypanosoma brucei. EMBO J 16: 7422-7431.

McCulloch R, Rudenko G, Borst P 1997. Gene conversions mediating antigenic variation in Trypanosoma brucei can occur in variant surface glycoprotein expression sites lacking 70-base-pair repeat sequences.
Mol Cell Biol 17: 833-843.

Munoz-Jordan JL, Davies K, Cross GAM 1996. Stable expression of mosaic coats of Variant Surface Glycoproteins in Trypanosoma brucei. Science 272: 1795-1797.

Navarro M, Cross GAM 1996. DNA rearrangements associated with multiple consecutive directed antigenic switches in Trypanosoma brucei. Mol Cell Biol 16: 3615-3625.

Rudenko G, Blundell PA, Dirks-Mulder A, Kieft R, Borst $P$ 1995. A ribosomal DNA promoter replacing the promoter of a telomeric VSG gene expression site can be efficiently switched on and off in T. brucei. Cell 83: 547-553.

Rudenko G, Chaves I, Dirks-Mulder A, Borst P 1998a. Selection for activation of a new variant surface glycoprotein gene expression site in Trypanosoma brucei can result in deletion of the old one. Mol Biochem Parasitol 95: 97-109.

Rudenko G, Cross M, Borst P 1998b. Changing the end: antigenic variation orchestrated at the telomeres of African trypanosomes. Trends in Microbiol 6: 113-117.

Van Leeuwen F, Wijsman ER, Kieft R, van der Marel GA, van Boom JH, Borst P 1997. Localization of the modified base $\mathrm{J}$ in telomeric VSG gene expression sites of Trypanosoma brucei. Genes Dev 11: 3232-3241.

Vanhamme L, Pays E 1995. Control of gene expression in trypanosomes. Microbiol Rev 59: 223-240.

Vassella E, Boshart M 1996. High molecular mass agarose matrix supports growth of bloodstream forms of pleomorphic Trypanosoma brucei strains in axenic culture. Mol Biochem Parasitol 82: 91-105. 
